\title{
Can Physical Activity Improve Depression, Coping \& Motivation to Exercise in Children and Youth Experiencing Challenges to Mental Wellness?
}

\author{
Scott Oddie, Denise Fredeen, Brandy Williamson, Drew DeClerck, Sacha Doe, \\ Kelly Moslenko \\ Red Deer College, Red Deer, Canada \\ Email: scott.oddie@albertahealthservices.ca
}

Received 23 October 2014; revised 18 November 2014; accepted 9 December 2014

Copyright (C) 2014 by authors and Scientific Research Publishing Inc.

This work is licensed under the Creative Commons Attribution International License (CC BY). http://creativecommons.org/licenses/by/4.0/

(c) (i) Open Access

\section{Abstract}

This study examined the influence of a physical activity (PA) program (Move Your Mood) on children and adolescents receiving services in community mental health clinics. Participants $(\mathbf{N}=35)$ were referred to the (PA) program by their mental health therapist. Coaches engaged participants in individual one-on-one and group activity sessions for eight weeks. Participant heart rates were monitored during physical activity sessions and designed to achieve moderate to high intensity. Participants reported significant improvements in mood immediately following physical activity. Measures of motivation to exercise, coping, and depression were taken before program participation, at 4-weeks, and at completion of the 8 week program. Results indicate that the PA program significantly improved child and adolescent ability to cope as well as their intrinsic motive to exercise. In addition, the PA program significantly reduced self-reported depressive symptoms. Qualitative analysis indicates that social supports and enhanced self-efficacy resulting from physical activity engagement and sessions are key factors associated with program outcomes. The current study provides evidence to support three key psychosocial theories: social interaction, distraction hypothesis, and mastery hypothesis.

\section{Keywords}

Physical Activity, Children, Youth, Mental Health, Community, Coping, Depression 


\section{Introduction}

Mental health affects how we feel, think, communicate and understand. In young people, mental health can affect schooling outcomes, social development, capacity to contribute to workforce and community, and suicide (Sheehan, Paed-Erbrederis, \& McLoughlin, 2000). With approximately 15\% of children and adolescents affected by a diagnosable mental illness (Waddell \& Shepherd, 2002), and 34\% of students in Grades 7 - 12 reporting symptoms of, depression, anxiety or social dysfunction (Paglia-Boak et al., 2012), it is clear that our children and adolescents are struggling to navigate life's challenges.

While the high number of children and adolescents struggling with a mental health problem is alarming, bestpractice has revealed that mental health can be improved not just by focusing on the problem but by enhancing positive factors (Joint Consortium for School Health, 2010). One positive factor that young people can change in their lives is their level of physical activity. People who exercise 2 - 3 times per week show lower levels of depression, anger, stress and other negative emotions than those who do not (Calfas \& Taylor, 1994). Adolescents who show higher physical activity levels are also more mentally resilient (Gerber, Kalak, Lemola, Clough, \& Brand, 2012). Furthermore, being physically active as a child can promote future mental wellness in adulthood. Despite its benefits, only 7\% of children and adolescents get the recommended level of 60 minutes of moderate to vigorous physical activity per day (Colley et al., 2011).

Physical activity and movement is an essential part of the healthy development of children and adolescents, and is acknowledged as being beneficial to maintain psychological well-being (Annesi, 2004). Sedentary behavior and low levels of physical activity participation during this time can result in behavioural and emotional challenges that can impact children and adolescents socially, emotionally, physiologically, and academically (Jerstad, Boutelle, Ness, \& Stice, 2010; Kantomaa, Tammelin, Ebeling, \& Taanila, 2008). The higher incidence of mental wellness challenges among this population and the potential long-term effects of having such issues during this period of development make the study of effective psychological interventions for children particularly important. It is also important to develop physical activity intervention that will change the exercise habits of children and adolescents to maintain health as physical activity can impact psychological wellness (Kantomaa et al., 2008) and physical activity engagement during adulthood (Prasad, St-Hilaire, Wong, Peterson, \& Loftin, 2009). Despite the clear need for research to study the effect of exercise on children and adolescents, the majority of research in this area focuses on adult participants (Annesi, 2004; Rothon et al., 2010).

In previous correlational studies, children who reported greater amounts of physical activity had lower levels of depression and anxiety, and higher levels of self-esteem and self-efficacy (Calfas \& Taylor, 1994). A crosssectional and longitudinal study by Rothon and colleagues (2010) found that every additional hour of physical activity children performed was associated with an $8 \%$ decrease in their odds of reporting depressive symptoms. Physical activity participation has also been associated with an improvement in children's coping mechanisms and lowered perceived stress (Kantomaa et al., 2008; Brown, Welsh, Labbé, Vitulli, \& Kulkarni, 1992). This would indicate that physical activity can improve resiliency and that physical activity could possibly be used as a preventative measure for those at higher risk of developing challenges to mental wellness.

There are several theories as to what the underlying mechanisms for the positive effects that physical activity has on psychological wellness, and there is yet to be clear consensus among researchers. The current theories fall within the physiological and psychosocial fields of study (Annesi, 2004). One physiologically-based theory proposes that it is due to the release of dopamine and beta endorphins during physical exercise, which has a relaxing effect (Stella et al., 2005). Other proposed physiological explanations for the effects include the changes to levels of endorphins, norepinephrine, serotonin, and core body temperature that result from engaging in physical activity (Annesi, 2004; Kantomaa et al., 2008). The psychosocial explanations for the effects include: distraction, task mastery, social interaction, and improving mood through the self-esteem gained from exercising (Annesi, 2004; Rothon et al., 2010).

A meta-analysis conducted by Stanthopoulou, Powers, Berry, Smits, \& Otto (2006) serves to show the effectiveness of physical activity on mental wellness with results suggesting health care providers integrate treatment options based on a biopsychosocial model. The biopsychosocial model focuses on caring and treating an individual on all dimensions, including physically and psychologically so that the mind and body are treated as one (Scherger, 2005). Effective programs should be based on this holistic approach by introducing children and youth who are facing difficulties in mental wellness to physical activity programs with the goal of improving their overall well-being. 
There is some debate as to which type of exercise (i.e., aerobic vs. anaerobic) has the greatest effects but the majority of the research indicates that aerobic activity is more effective in reducing symptoms associated with depression and anxiety (Azar, Ball, Salmon, \& Cleland, 2008; Richardson et al., 2005). Aerobic activity is said to be more effective because it produces an effect that is similar to that of an antidepressant. There has been no identification of a standard or preferred physical activity session as previous studies that have investigated physical activity interventions and mental health were not uniform in design and therefore varied in form, duration, and intensity of physical activity sessions (Larun, Nordheim, Ekeland, Hagan, \& Heian, 2009). While the Canadian Physical Activity Guideline recommends children engage in 60 minutes of moderate to vigorous physical activity a day, most interventions were effective when participants were engaged in physical activity sessions that were of moderate to vigorous intensity for 30 to 45 minutes in duration, for 3 - 5 days a week for a period of 12 weeks (Calfas \& Taylor, 1994; Richardson et al., 2005; Strong et al., 2005).

Research has also suggested key areas that a physical activity programs should focus upon. Whitelaw, Teuton, Swift and Scobie (2010) emphasize the known association between physical activity and mental health in young people and have suggested a shift from trying to understand why there is a link to how we best deliver interventions to improve wellness. Further, Whitelaw et al. (2010) outline key components that physical activity programs include. A "whole system/multi-sector approach" should be used that includes schools, health services and community based delivery. Ease of access to high quality and safe activity opportunities and facilities was recommended. In relation to activity itself, the ability to experience a range of types (e.g., endurance, flexibility and strength training and, individual and group opportunities) based on choice and individually determined goals. Orientation of physical activity provision was seen as preferably associated with positive and enjoyable experiences, decreased pressure with success based on an immediate sense of accomplishment, rather than "winning" or long-term health benefits. There was also a perception that activity should attempt to foster psychological competencies (e.g., control, autonomy, and self-efficacy). Peer models that foster participation activities that children and youth can enjoy with family and friends. Finally it was suggested that interventions should be delivered by high quality teachers/coaches and (local) leaders.

The Move Your Mood (MYM) study was designed with these recommendations in mind. The primary purpose of this study was to integrate physical activity into current treatment programs for children and adolescents accessing mental health supports in their community with an aim to improve mental wellness. This was done using an 8 week physical activity program that monitored quantitative indicators of depression, coping, and perceptions of physical activity as well as self-reported indicators of mood and satisfaction. This study hypothesized that mental wellness in children and adolescents would improve as a result of participation in the MYM physical activity program and that participant's attitudes and perceptions of physical activity would be enhanced.

\section{Methods}

\subsection{Participants}

Program Intervention Group. Participants were children and adolescents aged 10 - 17 in central Alberta $(N=35$; 11 males; 24 females). All participants were facing challenges to mental wellness and were seeing a therapist in a community-based clinical setting at the time of the study. Clinical diagnoses (i.e., depression) were not made available to the research or program team members. Incentives were awarded to encourage participation and included a $\$ 50$ gift card to a health/fitness related business at four weeks and an iPod touch or gift card of equal value to a health/fitness related business at eight weeks. Additionally some of the participants received a one year city pass to recreation facilities if they indicated they were a Move Your Mood participant. Bus passes were also provided to participants who use that form of transportation to access community sites where physical activity sessions occurred. Parents/caregivers of participants received a \$25 gas card at intake, week two, week four and week six to support transportation of participants to physical activity (PA) sessions.

\subsection{Procedure}

Recruitment. Participants were recruited to the MYM program by referral of their therapist. Following referral, the MYM Project Lead or Coaches scheduled appointments with the adolescents and their parents where informed consent (including parental consent for youth under 16 years of age) was provided. Once informed consent was obtained, participants were screened to determine if there were any pre-existing medical conditions that 
put them at risk for injury during PA sessions using an online Physical Activity Readiness Questionnaire (PARQ). The PAR-Q is a 7 item questionnaire to determine the presence of pre-existing health concerns that would require the participant to seek medical advice before participating in the PA sessions (Shephard, 1988; Thomas, Reading, \& Shephard, 1992). Any concern that was identified would be recommended to seek doctor approval or an exercise professional before engaging in any PA (this was not the case for any of the participants in this study). Participants then completed an online questionnaire that contained the Behavioural Regulation in Exercise Questionnaire, the Coping Efficacy Scale, and the Depression Self-Rating Scale for children and adolescents. This online questionnaire was completed at intake, week four, and again at week eight. Additionally, a focus group with participants was held upon completion of the eight week program to receive feedback on the MYM program. This study received approval from the Red Deer College Research Ethics Board.

Physical Activity Sessions. The PA sessions occurred two to three times per week and were designed to provide thirty minutes at a moderate to vigorous intensity (as determined by heart rate monitors worn by participants during activity sessions). One of the PA sessions each week was led by a MYM Coach in a one-on-one setting. The one-on-one sessions were tailored to the individual participant's interests and therefore varied in the manner of which they were conducted (i.e., floor hockey, in door rock-climbing, weight training) but were 30 60 minutes in duration. There was an additional group PA session each week held in a dance studio. The group session was an hour in duration and also varied in form (i.e., circuit training, hip-hop dance, yoga, cross fit) from week to week. The first and last ten minutes were typically designated for warm up and cool down stretching. Finally, participants were asked to complete at least one additional at home PA session for a minimum of thirty minutes. Participants were enrolled in one of three MYM programs (10 - 12 participants per program) that began in January, March or May 2013. This reduced possible seasonal bias effects that may have influenced participant responses. Pre- and post-program responses were collected from December through July.

\subsection{Measures}

Demographics. Age, gender, and location of activities were collected in order to obtain a basic understanding of participants and activity environments.

Heart Rate. A random sample of three to four participants in the group PA sessions along with participants in the one-on-one sessions wore heart rate monitors (Scosche RYTHYM) in order to determine the level of intensity reached during the PA sessions. The heart rate monitors were linked to the iPads via Bluetooth in which each PA session was recorded and measured durations, beats per minute (bpm), and kilocalories burned during activity sessions. For the purposes of this study, only the bpm were utilized to determine the duration and intensity (i.e., moderate to vigorous) of physical activity.

Self-Reported Mood. A mood scale was created on a 10-point Likert scale ( 1 = not happy to $10=$ very happy) to measure changes in self-reported mood. Participants recorded their mood before and immediately following the PA sessions to determine if there were any effects on self-reported mood status.

Behavioural Regulation in Exercise Questionnaire-2 (BREQ-2). The BREQ-2 is a 19-item self-report questionnaire that is used to evaluate the motivating factors that children and adolescents have about exercise (Markland \& Tobin, 2004). There are 5 subscales which distinguish between different types of exercisers. Amotivated individuals do not have any intention to engage in exercise and include such questions as "I don’t see why I should have to exercise" (Chronbach's $\alpha=0.83$ ). Children who exercise because of external influences or rewards (i.e., "I exercise because other people say I should") are externally motivated (Chronbach's $\alpha=0.79$ ) while those who exercise because they enjoy it (i.e., "I exercise because it's fun") are intrinsically motivated (Chronbach's $\alpha=0.86$ ). Introjected individuals do not necessarily enjoy exercise but partake in it to reap its benefits (i.e., "I feel guilty when I don't exercise"; Chronbach's $\alpha=0.80$ ) and finally, identified individuals exercise because they view it as important and a way to achieve valued outcomes (i.e., "I value the benefits of exercise"; Chronbach's $\alpha=0.80)$. Along with the five subscales there is an overall measure called the Relative Autonomy Index (RAI) or, the Self-Determined Motivation score (Gillison, Standage, \& Skevington, 2006). This score measures the degree to which individuals are intrinsically motivated to exercise on a dimension of -24 to 20. Higher positive scores on this dimension reflect a more intrinsic motivation while lower scores are indicative of being less intrinsically motivated to be physically active.

The Coping Efficacy Scale (CES). The Coping Efficacy Scale is a 7-item scale that assesses how well children and adolescents believe they can independently manage difficult situations (Sandler, Tein, Mehta, Wolchik, \& 
Ayers, 2000). The scale evaluates how the individual has dealt with difficult situations in the past (e.g., "overall, how satisfied are you with the way you handled your problems during the last month?”) and how they think they will cope with similar situations in the future (e.g., "in the future, how good do you think that you will usually be in handling your problems?"). It uses four point Likert scales that range from "not at all satisfied", "did not work at all", and "not at all good" (1) to "very satisfied", "worked very well", and "very good" (4). A high score indicates that the individual has strongly held beliefs in their effectiveness and ability to deal with difficult situations while a lower score may indicate that the individual feels that they are unable to deal with difficult situations or that they do not engage in adequate coping strategies (Chronbach $\alpha$ 's from 0.82 to 0.91 ).

The Depression Self-Rating Scale for Children (DSRS). The DSRS for children is an 18-item standardized questionnaire that uses a three-point Likert scale $(1=$ never to $3=$ mostly $)$ and the total score is structured to identify depression in children (Birleson, 1981; $\alpha=0.80$ ). Questions such as "I feel like crying", "I like to go out to play", and "I feel very lonely" are used to distinguish depressed from non-depressed individuals. Depression in this questionnaire is defined by the Diagnostic and Statistical Manual of Mental Disorders classification (DSM-IV) and while the questionnaire itself is not sufficient for a clinical diagnosis, individuals who have a score of 15 or higher are more likely to be depressed. Participants' scores over 15 were brought to the attention of their therapist.

Qualitative Evaluation. A focus group was held upon completion of the eight weeks in which open ended questions were asked of the participants in order to evaluate the MYM program. Questions such as "what did you like about the program and why", "what would you change about the program and why", and "what did you learn about the program about the connection between movement and mood" were asked. The focus group served to identify strengths and potential areas for improvement associated with the MYM program; to determine if there were any individual changes in perspective towards physical activity and mental wellness; and to gain a better understanding as to how the participants planned to engage in physical activity after the MYM program.

\section{Results}

\subsection{Intensity of Program Activity}

Data from heart rate monitors was analyzed to determine if the duration of moderate to vigorous activity sustained during the PA sessions met the goal of 30 minutes. Nineteen samples were selected randomly and the average time spent in moderate to vigorous intensity was 29.02 minutes. Moderate to vigorous intensity was based on heart rate and included the range of 140 - $200 \mathrm{bpm}$ (Virtanen, 2011).

\section{Self-Reported Mood}

Self-reported mood recorded by participants just prior to, and immediately after PA sessions increased significantly from $5.8 \pm 0.3( \pm \mathrm{SEM})$ to $7.3 \pm 0.2(\mathrm{t}(34)=10.8, p<0.0001)$.

\subsection{Motivation to Exercise, Coping, and Depression}

A Shapiro-Wilk test was completed along with a measure of skewness and kurtosis to ensure the assumption of normality was being met. All three measures were not significant and therefore, parametric testing was used to analyze the data. A repeated measures ANOVA was conducted to determine if there were any changes in participants' perceptions of their motivation to exercise, coping skills, and self-rated depression scores from week 1 to week 4 and to week 8.

Figure 1 shows the participants' responses to the motivation, coping and depression measures before, after 4 weeks of program participation, and at the conclusion of the 8 week program. Analysis indicated significant improvements in participants' motivation to exercise $(\mathrm{F}(2,33)=3.5, p<0.04)$ and ability to cope $(\mathrm{F}(2,33)=15.8$, $p<0.0001)$. There was also a significant decrease in self-reported depression scores $(\mathrm{F}(2,33)=16.7, p<$ $0.0001)$.

\subsection{Focus Group Themes}

Qualitative analysis found that participants were extremely satisfied with the Move Your Mood program. The qualitative data was analyzed using grounded theme analysis. Two individual coders were utilized to analyze the 


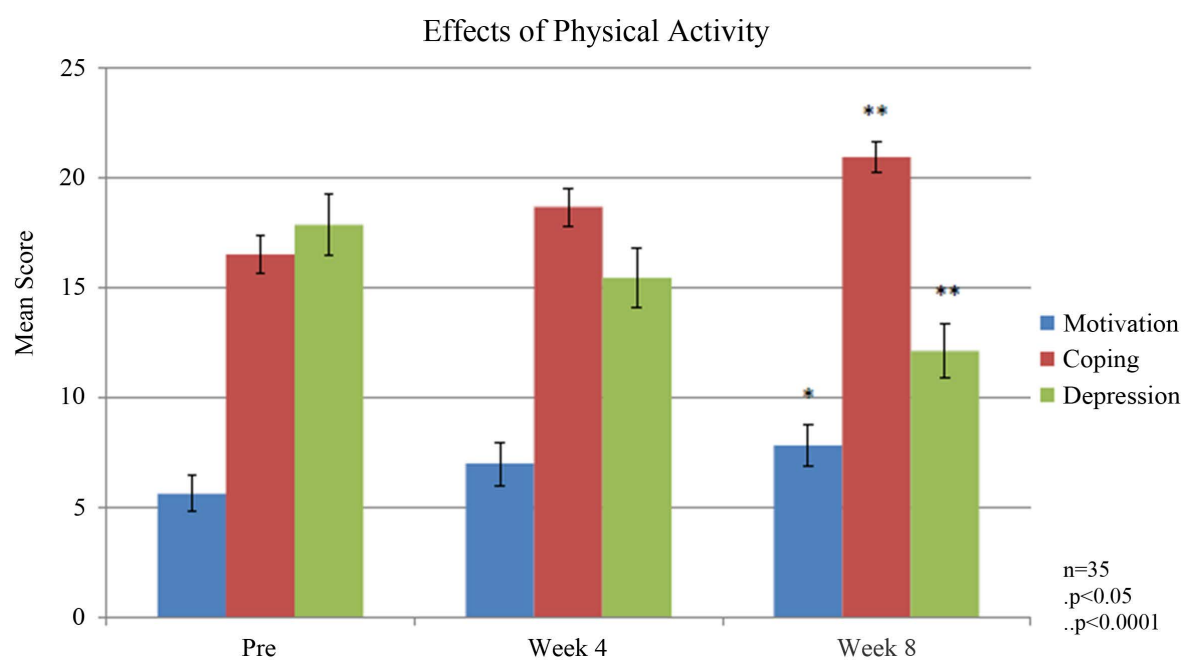

Figure 1. The effect of the physical activity program on measures of participants' self-reported motivation to exercise, coping with stress and depression. Motivation to engage in physical activity increased significantly during the 8-week PA Program. Ability to cope with stress increased significantly and self-reported depression decreased significantly.

data in which they came to a consensus on the themes depicted in the focus groups. The feedback received from the participants was valuable in establishing the benefits of the MYM program as well as potential barriers participants felt would prevent them from being active in the future.

Social Interaction. Social interaction was the most predominant theme depicted in both of the focus group sessions. When asked what they liked about the program the majority of responses included answers such as: "I really like the exercise but what made it more fun was when you could do it with friends and making new friends," "getting to know people," and "getting to hang out with friends". The participants did not know each other prior to entering the MYM program so it should be noted that the social interactions were newly established upon entering the program. This is an interesting aspect to further investigate as it may indicate the significance of any type of social interaction (i.e., new vs. established social connections) when it comes to enhancing mental wellness.

Social Support. A majority of the participants also commented on the level of support they received, not only from the group but from the MYM coaches as well. Responses such as "just having the group around helps a lot" and "you get to see people and then you excel" are indicative of social support provided by the group while responses such as "the coaches... are always telling you how good you're doing" and "[the coaches] are someone you can talk to if you need to" indicate the support provided by the MYM coaches in the group and one-on-one sessions.

Improved Mood. Several participants also identified that their moods had improved following the PA sessions which was indicated by comments such as "I know no matter what I will always feel a little better, even if it's a little difference or big difference I try to focus on that because it's better than feeling crappy", "Some days I'd be like angry like before I would go and then I would go to the activity and then as soon as I would get back I would be really happy" and "I felt happier after". These responses provide evidence that PA has an immediate effect on enhancing mood.

Education. A large number of participants seemed to benefit from the educational properties of the MYM program. This was seen in responses such as "I was able to learn a lot of stuff and now I can work out by myself at home", "[most valuable part] was learning how to use the proper equipment and how to use it right", "learning the different exercises and how hard you actually have to do them to make it worth it [was most valuable", and "I've learned a lot more from [MYM], I guess I feel like I've gained something". This provides evidence indicating that the MYM program provides participants with the proper information needed to pursue PA in the future independently.

Confidence. Another theme discovered was that the MYM instilled a sense of confidence in the participants in regards to working out that they did not have prior to MYM. A large majority of participants commented in re- 
gards to their newly established levels of confidence. For example, "[the] group built up confidence to work out like by yourself in public whereas if I do it it's not something I would have been entirely comfortable with", "I can actually go out and do stuff now that I couldn't do before", and "I don't care [what anyone says] anymore, I'm going to try and do this for me" indicate that MYM gave participants the confidence they needed to work out on their own.

Motivation. The theme of motivation was mainly found in regards to the coaches but it was a predominant theme that was discussed by a majority of the participants indicating its importance. Responses supporting this include: "[coaches] pushed us hard but it was good though", they push you past your limits sometimes but it's a good thing", and "I liked [the coaches], they were nice, they pushed you to your limits but that's a good thing".

Stress Reduction. An additional benefit participants identified was that the MYM program reduced the amount of stress they were feeling. Most participants found this benefit to be quite obvious which is seen in statements such as "usually when I show up I'm usually all stressed out and then when I get moving it's gone" and "it makes you less stressed".

Distraction. Several participants also indicated that a valuable benefit of the MYM program was the distraction from life it provided. Participants made comments such as "[MYM] got my mind off a lot of stuff", "the program for me, it took my mind off of a lot of things that were happening with family and friends", and "takes your mind off it" that support the theme of distraction.

Improved Sleep. Many participants identified with this theme in that they indicated that the MYM program has improved their sleep habits. This was found in the following responses: "I don't sleep very well and after I actually sleep so that did help and I could actually get up the next morning" and "I would come home and my mom would ask me how was it and I was like eh, it was okay and then I would like fall asleep so fast”.

Increased PA and Opportunities. When discussing the most valuable aspect of MYM and what participants enjoyed the most, a recurring theme was that of increased activity along with opportunities to explore new avenues in the realm of PA. This was seen in responses such as "it gives you opportunities to try different things", "[I liked] being active" and "[I] liked the variation, I was expecting them to be teaching it to us but then they brought in their own instructors so it was interesting and kind of changed it up and then it also caused us to learn more things and we got introduced to those programs”.

Barriers. The focus groups also addressed potential barriers that will prevent the participants from maintaining engagement in PA in the future. The barriers discovered were time, "making the time to do it" and "finding the time"; motivation "finding the motivation [to continue]" and "because it's not structured this day and this time I'm going to put it off"; and finally transportation "getting to the place to do it".

\section{Discussion}

The purpose of this present study was to determine the effect PA has on children and adolescents facing challenges to mental health. The findings of this study are consistent with our hypothesis indicating that PA in children and adolescents of a moderate intensity for 2 - 3:30 minute periods per week is linked to an improvement in overall mental wellness. Self-reported mood increased significantly immediately after participating in physical activity sessions. Participants experienced a significant increase in perceived motivation to exercise and ability to cope with stress and a significant decrease in self-reported depression scores.

Consistent with previous research (Prasad et al., 2009; Rothon et al., 2010; Strong et al., 2005; among others) our results indicate that physical activity is an effective intervention in reducing the symptoms that are associated with challenges to mental wellness (e.g., depression, anxiety). Although there were no confirmed clinical diagnoses amongst our participants, the DSRS tool assesses three aspects of depression (low mood, negative thinking, and reduced activity). Given that this is a reliable and validated measure it can be inferred that it is efficacious in measuring characteristics associated with depression (Birleson, 1981). Our results; therefore, can be interpreted as an improvement in the symptomology of depression rather than an improvement in the clinical diagnosis of depression itself. Additionally, the results indicate that individuals need to be engaged in physical activity for a minimum of 4 weeks before there are any noticeable decreases in symptomology.

There are no well-established mechanisms that explain the changes in mental wellness one experiences following PA, however, physiological explanations are widely implied (Hamer, Endrighi, \& Poole, 2012; Vilhjalmsson \& Thorlindsson, 1992). The acceptance of physiological mechanisms typically leads to an exclusion of other psychosocial based hypotheses. One of these psychosocial theories is based on social interaction and the 
indirect effects PA has on mood by means of increased access to social factors (Rothon et al., 2010). This theory postulates that the increased opportunities for social interaction, along with the organized format of the group PA sessions (Vilhjalmsson \& Thorlindsson, 1992), lead to an improvement of mood. While the research supporting this theory is rather limited it does merit some investigation in regards to our results. We did not measure social interaction or support directly but it became evident as influencing factors from our qualitative data. Both social interaction and social support emerged as predominant themes (i.e., "what made it more fun was when you could do it with friends and making new friends") suggesting that increased interaction within a social setting and the creation of new friendships was an important factor in the improvements in well-being participants experienced.

Additionally, there has been support found in previous research to suggest that organized group activities enhance the positive aspects of well-being. The design of the current study allowed for this as participants engaged in group PA sessions (Vilhjalmsson \& Thorlindsson, 1992). Again, support for this was found within our qualitative data in which participants acknowledged the importance of the group activities and how it affected them in a positive manner (i.e., "you get to see people (in the group) and excel”). The evidence presented conforms well to the social interaction theory but can only be used as speculative analysis as social factors were not directly measured or controlled for.

Two additional psychosocial theories utilized to explain the connection between PA and depression would presumably have a more immediate impact. These theories include the distraction hypothesis which says, it is the escape from life PA affords that accounts for increases in mood; and the mastery hypothesis which suggests that it is the completion of a new task that evokes a sense of achievement within an individual which in turn improves mood (Jerstad et al., 2010; Lawlor \& Hopker, 2001; Rothon et al., 2010). The mood scales that were completed by participants before PA sessions and immediately following PA sessions may be indicative of these theories. As the average mood of participants significantly increased following PA sessions, the immediacy of these proposed hypotheses appears to be somewhat appropriate in inferring the presence of a mechanism that is responsible for producing instantaneous effects following PA sessions. The notion of immediacy is especially evident for the distraction hypothesis which obtained support within our qualitative data and emerged as a predominant theme. Many of the participants stated that the PA sessions provided them a distraction to problems they were facing as it provided a means to take their mind off these issues. While the research on this proposed hypothesis is rather limited, it does warrant further investigation as the current study provides strong evidence in its favour.

The current study also examined the effects that PA had on one's perceived ability to cope and motivation to exercise. These two variables both showed significant improvements. Participant's perceived abilities to cope with future stressors and intrinsic motivation significantly improved across the eight week PA program. One of the most comprehensive explanations of this finding is the concept of self-efficacy which is the personal belief an individual has in regards to his or her ability to complete a designated task (Bandura, 1977). In a reciprocal action, completion of the PA sessions creates a sense of achievement within the participants which, in accordance to the aforementioned mastery hypothesis, enhances one's self-efficacy. This increase in self-efficacy further motivates the participants to engage in additional PA sessions. Once stronger self-efficacy expectations have been established, a shift in motivation takes place as the participants have established stronger beliefs in their own abilities. Based on this reasoning, the significant improvement in the RAI scores after completion of the MYM program (calculated from the BREQ-2) can potentially be attributed to an increase in self-efficacy which has been established by the learning and completion of the PA sessions.

The concept of self-efficacy can also be utilized when addressing the results of the coping measures. According to Bandura (1977), once self-efficacy is established it can generalize to a broad range of situations not limited to the environment in which it was created. This proposes that although self-efficacy may be established within the context of the PA sessions it can be extended to additional areas allowing for improvement in participant's perceived ability to cope. This prospective explanation of results is supported by research conducted by Richardson et al. (2005) who state that incremental achievement of PA will gradually increase levels of self-efficacy. The structure of the current study allows for incremental achievement to take place as participants engage in PA sessions weekly. Upon intake, when participants complete their first online questionnaire, self-efficacy levels would likely be relatively low as they are entering a new environment with new tasks which accounts for the lower scores on the CES at week one. As seen in the results, coping scores begin to improve after four weeks of PA sessions which is presumably because a greater level of self-efficacy had been established. The participants had instilled in themselves a stronger belief system in which they would be better suited to cope with fu- 
ture problems. Further research in the area of motivation to exercise and perceived ability to cope is required to infer these results as being related to PA and to provide evidence in support of self-efficacy being a driving force in the changes.

There were some limitations discovered in previous studies that the MYM program addressed giving the current study several strengths. One of the most prevalent limitations noted from a review of the literature was a subjective evaluation of PA provided by the participants as opposed to objective measures (Richardson et al., 2005). As Richardson and associates (2005) point out, self-monitoring of PA is difficult to do and less reliable than objective assessments; however, there are easy and efficient ways in which this can be overcome. One suggestion is through the use of heart rate monitors which measures intensity level by means of heart rate. This was an advantage to our study as heart rate monitors were utilized allowing us to gauge the level of intensity as well as the duration as to which participants remained in the target zone of moderate-vigorous. Having the heart rate monitors allowed for greater control over the quality of PA the participants were receiving as the MYM Coaches could visually determine if participants were in the appropriate intensity level to ensure they reached and maintained the target heart rate zones of 140 - 200 bpm (Calfas \& Taylor, 1994; Virtanen, 2011). However, the average time spent in moderate to vigorous activity by participants in this study was 29 minutes even though the intention was to set a minimum threshold of at 30 minutes for each session. This suggests that coaches may not be able to accurately determine physical activity levels visually and may need to establish better indicators such as heart rate monitors.

Another concern identified in previous studies was the use of measurement tools that were tailored to adults instead of children (Kantomaa et al., 2008; Motl, Birnbaum, Kubik, \& Dishman, 2004). Using tools designed for adults when measuring depressive symptoms in children presents two important problems. One, because adults and children experience depression and its associated symptoms differently a measure designed for adults may not accurately identify depressive symptoms in children. This in turn prevents generalization to a larger population because it is not a validated measure for the sampled age group. The current study used assessment tools that were reliable, validated, and designed for children allowing for a more comprehensive study that was specifically tailored to them.

\section{Limitations and Future Directions}

Despite the promising results of the current study, there are some limitations that need to be addressed. The absence of a control group prohibits the comparison of a PA based intervention to other methods of treatment (i.e., therapy, medication). Children and youth in this study were referred by their therapists in community health clinic settings. This limitation is restrictive in the sense that it does not allow the ability to determine whether or not PA based interventions are more effective than traditional treatment methods. Physical activity interventions are desirable because they are less expensive and have little to no side effects; however, due to the absence of a control group it cannot be recommended that an individual undergoing more traditional methods of treatment switch to this potentially more enticing intervention. The lack of control group was not a flaw in the design as potential participants were given the opportunity to participate in a control group if they did not want to engage in the PA sessions. All the individuals referred to the MYM program chose to be in the intervention group and due to time restrictions it was not feasible to have a wait list control group.

Another limitation associated with this study was the lack of any clinical diagnoses. Due to therapist/client confidentiality we were unable to obtain any formal diagnoses of our participants. This threatens internal validity as we cannot generalize the results to the clinically depressed and anxiety disordered population. Although the DSRS can measure certain characteristics of depression, alone it cannot be used as a diagnostic tool to determine mental disorder and thus limits the generalizability of the results. Clinical psychologist assessment alongside the use of validated tools is needed to confirm psychological disorders.

Lastly, the influence of social interaction/support was a potential confounding variable which makes it difficult to assess the true influence that physical activity has on the dependent variables collected here. As previously discussed, social interaction is a mechanism that can be used to explain the effects PA has on mental wellness (Rothon et al., 2010). Additionally, a predominant theme within our qualitative data was that of social support and interaction. Both pieces of information provide complementary evidence in supporting a strong influence of social support and interaction. The presented evidence is reason for future studies to include a measure of social interaction/support in order to validate PA, not social factors, as being an effective intervention for 
improving mental wellness.

Based on the wide array of positive outcomes PA affords, future studies would benefit from the inclusion of additional measures. As previously mentioned, including a measure of social interaction/support is imperative to determine the degree of influence these two factors have in regards to improvement in mental wellness. It would also be beneficial to include participant's academic achievements as a measure. Previous research has shown that PA can improve academic ability and so measuring this would be of a great benefit especially to the targeted age group of adolescents (Sibley \& Etnier, 2003).

Finally, the PA sessions should continue in the manner of group and one-on-one sessions. Social support and interaction appear to be important determinants influencing the outcomes of PA interventions which are provided in both PA settings as determined from the qualitative data. In the current study, participants were asked to complete one at home activity which was self-reported and as such, is not a reliable measure. One suggestion identified in the focus groups was to allow the option of participating in two group sessions a week opposed to just one. This would provide all three required PA sessions to be objectively measured, via heart rate monitors, and would ensure all participants were engaging in equal amounts of PA.

\section{Conclusion}

This study suggests that there is an association between physical activity and mental wellness in adolescents. Children and adolescents with challenges to mental wellness (receiving therapy in community mental health clinics) reported significant improvements in mood immediately following physical activity. Results also indicate that an eight week program that delivers physical activity, at moderate to high intensity for three 30 minute sessions per week, can significantly improve child and adolescent ability to cope as well as their intrinsic motive to exercise. In addition, the PA program significantly reduced self-reported depressive symptoms. The exact mechanisms as to which of these changes can be attributed to are still unclear but the current study provides evidence to support three key psychosocial theories: social interaction, distraction hypothesis, and mastery hypothesis.

\section{References}

Annesi, J. (2004). Relationship between Self-Efficacy and Changes in Rated Tension and Depression for 9- to 12-yr.-Old Children Enrolled in a 12-wk. After-School Program. Perceptual and Motor Skills, 99, 191-194. http://dx.doi.org/10.2466/pms.99.1.191-194

Azar, D., Ball, K., Salmon, J., \& Cleland, V. (2008). The Association between Physical Activity and Depressive Symptoms in Young Women: A Review. Mental Health and Physical Activity, 1, 82-88. http://dx.doi.org/10.1016/j.mhpa.2008.09.004

Bandura, A. (1977). Self-Efficacy: Toward a Unifying Theory of Behavioral Change. Psychological Review, 84, $191-215$. http://dx.doi.org/10.1037/0033-295X.84.2.191

Birleson, P. (1981). The Validity of Depressive Disorder in Childhood and the Development of a Self-Rating Scale: A Research Report. Journal of Child Psychology and Psychiatry, 22, 73-88. http://dx.doi.org/10.1111/j.1469-7610.1981.tb00533.x

Brown, S., Welsh, M., Labbe, E., Vitulli, W., \& Kulkarni, P. (1992). Aerobic Exercise in the Psychological Treatment of Adolescents. Perceptual and Motor Skills, 74, 555-560. http://dx.doi.org/10.2466/pms.1992.74.2.555

Calfas, K., \& Taylor, W. (1994). Effects of Physical Activity on Psychological Variables in Adolescents. Pediatric Exercise Science, 6, 406-423. http://journals.humankinetics.com/pes

Colley, R. C., Garriguet, D., Janssen, I., Craig, C. L., Clarke, J., \& Tremblay, M. S. (2011). Physical Activity of Canadian Children and Youth: Accelerometer Data from the 2007 to 2009 Canadian Health Measures Survey. Statistics Canada, Health Reports, 22. http://www.statcan.gc.ca/pub/82-003-X/2011001/article/11397-eng.htm

Gerber, M., Kalak, N., Lemola, S., Clough, P. J., Puhse, U., Elliot, C., Holsboer-Trachsler, E., \& Brand, S. (2012). Adolescents' Exercise and Physical Activity Are Associated with Mental Toughness. Mental Health and Physical Activity, 5, 3542. http://dx.doi.org/10.1016/j.mhpa.2012.02.004

Gillison, F. B., Standage, M., \& Skevingon, S. M. (2006). Relationships among Adolescents’ Weight Perceptions, Exercise Goals, Exercise Motivation, Quality of Life and Leisure-Time Exercise Behaviour: A Self-Determination Theory Approach. Health Education Research, 21, 836-847. http://dx.doi.org/10.1093/her/cyl139

Hamer, M., Endrighi, R., \& Poole, L. (2012). Physical Activity, Stress Reduction, and Mood: Insight into Immunological Mechanisms. Methods in Molecular Biology, 934, 89-102. http://dx.doi.org/10.1007/978-1-62703-071-7_5 
Joint Consortium for School Health (2010). Schools as a Setting for Promoting Positive Mental Health: Better Practices and Perspectives. http://www.jcsh-cces.ca/upload/JCSH\%20Best\%20Practice_Eng_Jan21.pdf

Jerstad, S., Boutelle, K., Ness, K., \& Stice, E. (2010). Prospective Reciprocal Relations between Physical Activity and Depression in Female Adolescents. Journal of Consulting and Clinical Psychology, 78, 268-272. http://dx.doi.org/10.1037/a0018793

Kantomaa, M., Tammelin, T., Ebeling, H., \& Taanila, A. (2008). Emotional and Behavioral Problems in Relation to Physical Activity in Youth. Medicine \& Science in Sports \& Exercise, 40, 1749-1756. http://dx.doi.org/10.1249/MSS.0b013e31817b8e82

Larun, L., Nordheim, L. V., Ekeland, E., Hagen, K. B., \& Heian, F. (2009). Exercise for Preventing and Treating Anxiety and Depression in Children and Young People. Cochrane Database of Systematic Reviews, 19, CD004691.

Lawlor, D. A., \& Hopker, S. W. (2001). The Effectiveness of Exercise as an Intervention in the Management of Depression: Systematic Review and Meta-Regression Analysis of Randomised Controlled Trials. British Medical Journal, 322, 763767. http://dx.doi.org/10.1136/bmj.322.7289.763

Markland, D., \& Tobin, V. (2004). A Modification to the Behavioural Regulation in Exercise Questionnaire to Include an Assessment of Amotivation. Journal of Sport and Exercise Psychology, 26, 191-196. http://journals.humankinetics.com/jsep

Motl, R. W., Birnbaum, A. S., Kubik, M. Y., \& Dishman, R. K. (2004). Naturally Occurring Changes in Physical Activity Are Inversely Related to Depressive Symptoms during Early Adolescence. Psychosomatic Medicine, 66, 336-342.

Paglia-Boak, A., Adlaf, E. M., Hamilton, H. A., Beitchman, J. H., Wolfe, D., \& Mann, R. E. (2012). The Mental Health and Well-Being of Ontario Students, 1991-2011. CAMH Research Document Series No. 34, The Centre for Addiction and Mental Health Website.

http://www.camh.ca/en/research/news_and_publications/ontario-student-drug-use-and-health-survey/Documents/2011\%2 0OSDUHS\%20Docs/2011OSDUHS Detailed MentalHealthReport.pdf

Prasad, A., St-Hilaire, S., Wong, M., Peterson, T., \& Loftin, J. (2009). Physical Activity and Depressive Symptoms in Rural Adolescents. North American Journal of Psychology, 11, 173-188. http://najp.8m.com/

Richardson, C., Faulkner, G., McDevitt, J., Skrinar, G., Hutchinson, D., \& Piette, J. (2005). Integrating Physical Activity into Mental Health Services for Persons with Serious Mental Illness. Psychiatric Services, 56, 324-331. http://dx.doi.org/10.1176/appi.ps.56.3.324

Rothon, C., Edwards, P., Kamaldeep, B., Viner, R., Taylor, S., \& Stansfeld, S. (2010). Physical Activity and Depressive Symptoms in Adolescents: A Prospective Study. BMC Medicine, 8, 32. http://dx.doi.org/10.1186/1741-7015-8-32

Sandler, I. N., Tein, J. Y., Mehta, P., Wolchik, S., \& Ayers, T. (2000). Coping Efficacy and Psychological Problems of Children of Divorce. Child Development, 71, 1099-1118. http://dx.doi.org/10.1111/1467-8624.00212

Scherger, J. E. (2005). The End of the Beginning: The Redesign Imperative in Family Medicine. Family Medicine, 37, 513516. http://www.stfm.org/NewsJournals/FamilyMedicine

Sibley, B., \& Etnier, J. (2003). The Relationship between Physical Activity and Cognition in Children: A Meta-Analysis. Pediatric Exercise Science, 15, 243-256. http://journals.humankinetics.com/pes

Sheehan, R., Paed-Erbrederis, C., \& McLoughlin, A. (2000). The ICARUS Project: Implications for Children with Adult Relatives under Stress. Clayton: Department of Social Work, Monash University. http://www.theicarusproject.net/

Shephard, R. J. (1988). PAR-Q, Canadian Home Fitness Test and Exercise Screening Alternatives. Sports Medicine, 5, 185195. http://dx.doi.org/10.2165/00007256-198805030-00005

Stanthopoulou, G., Powers, M., Berry, A., Smits, J., \& Otto, M. (2006). Exercise Interventions for Mental Health: A Quantitative and Qualitative Review. Clinical Psychology: Science and Practice, 13, 179-193. http://dx.doi.org/10.1111/j.1468-2850.2006.00021.x

Stella, S., Vilar, A., Lacroix, C., Fisberg, M., Santos, R., Mello, M., \& Tufik, S. (2005). Effects of Type of Physical Exercise and Leisure Activities on the Depression Scores of Obese Brazilian Adolescent Girls. Brazilian Journal of Medical and Biological Research, 38, 1683-1689. http://dx.doi.org/10.1590/S0100-879X2005001100017

Strong, W. B., Malina, R. M., Blimkie, C. J., Daniels, S. R., Gutin, B., Hergenroeder, A. C., Trudeau, F. et al. (2005). Evidence Based Physical Activity for School-Age Youth. Journal of Pediatrics, 146, 732-737. http://dx.doi.org/10.1016/j.jpeds.2005.01.055

Thomas, S., Reading, J., \& Shephard, R. J. (1992). Revision of the Physical-Activity Readiness Questionnaire (PAR-Q). Canadian Journal of Sport Sciences, 17, 338-345. http://www.researchgate.net/journal/0833-1235_Canadian_journal_of_sport_sciences_Journal_canadien_des_sciences_du _sport

Vilhjalmsson, R., \& Thorlindsson, T. (1992). The Integrative and Physiological Effects of Sport Participation: A Study of Adolescents. The Sociological Quarterly, 33, 637-647. http://dx.doi.org/10.1111/j.1533-8525.1992.tb00148.x 
Virtanen, P. (2011). Measurement of Children’s Physical Activity. Development of Polar Active Activity Monitor. Proceedings from the International Congress on Enhancement of Physical Activity of Children and Youth, Vuokatti, 7-9 April 2011.

Waddell, C., \& Shepherd, C. (2002). Prevalence of Mental Disorders in Children and Youth. Retrieved Government of British Columbia Ministry Children \& Family Development. http://www.mcf.gov.bc.ca/mental health/pdf/02a cymh.pdf

Whitelaw, S., Teuton, J., Swift, J., \& Scobie, G. (2010). The Physical Activity-Mental Wellbeing Association in Young People: A Case Study in Dealing with a Complex Public Health Topic Using a "Realistic Evaluation” Framework. Mental Health and Physical Activity, 3, 61-66. http://dx.doi.org/10.1016/j.mhpa.2010.06.001 
Scientific Research Publishing (SCIRP) is one of the largest Open Access journal publishers. It is currently publishing more than 200 open access, online, peer-reviewed journals covering a wide range of academic disciplines. SCIRP serves the worldwide academic communities and contributes to the progress and application of science with its publication.

Other selected journals from SCIRP are listed as below. Submit your manuscript to us via either submit@scirp.org or Online Submission Portal.
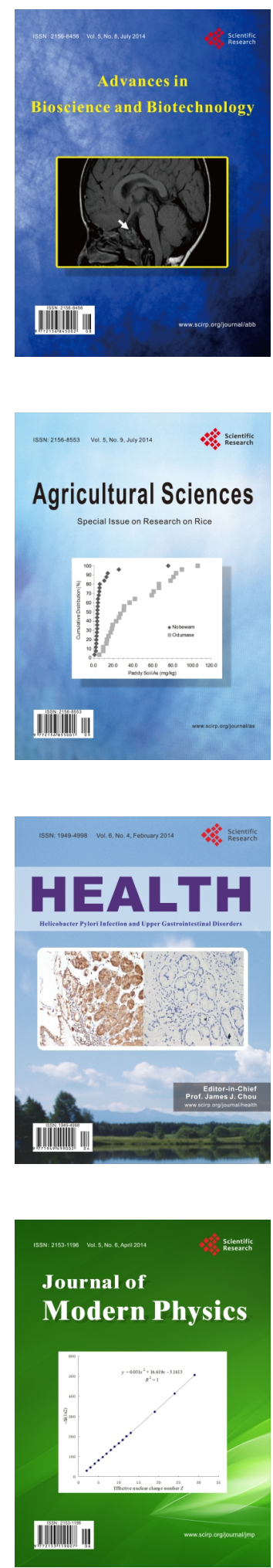
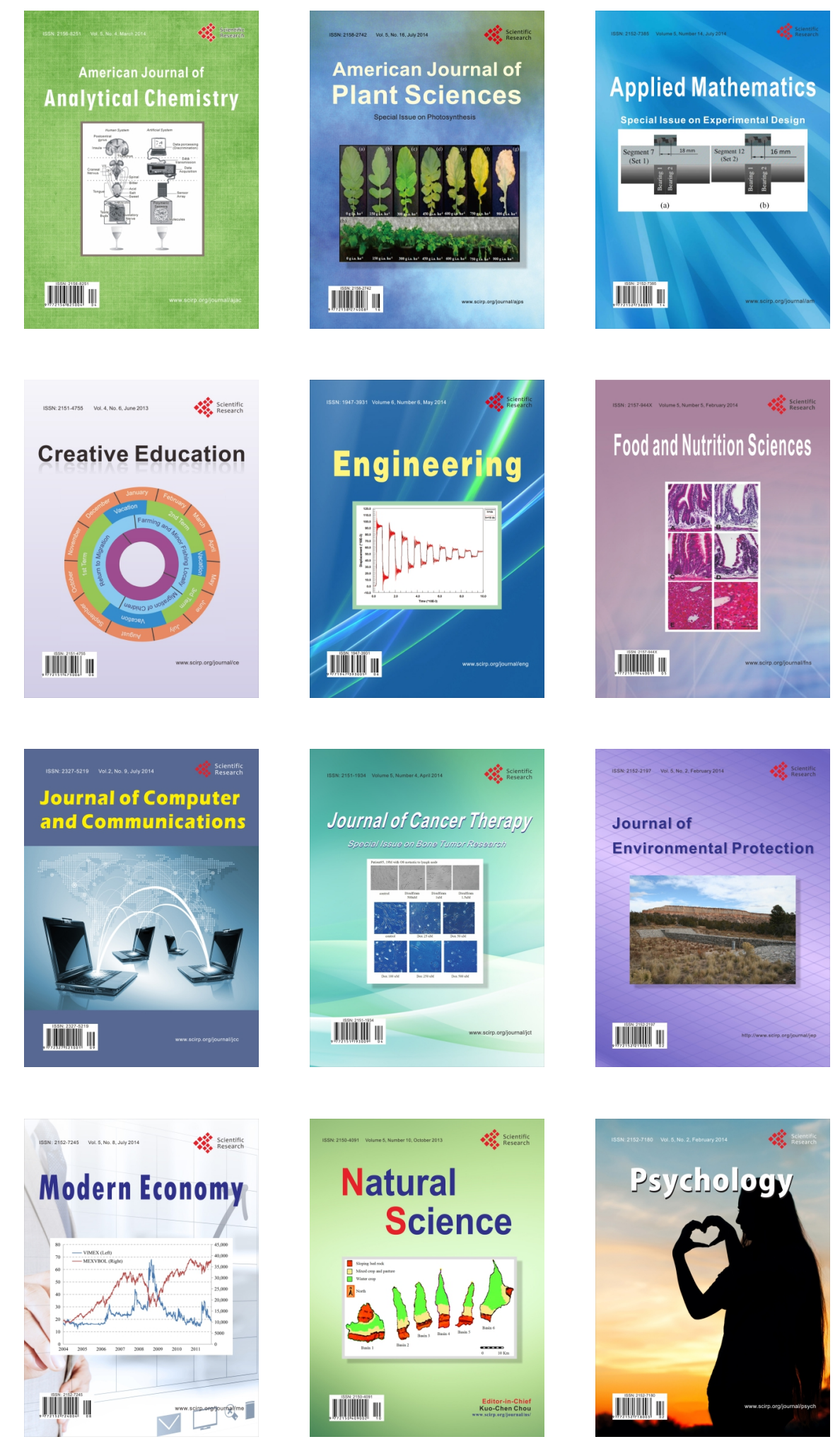